\title{
Cytokines: Pulling the Body Together as a Whole
}

\author{
Giamila Fantuzzi \\ Department of Kinesiology and Nutrition, University of Illinois at Chicago, \\ Chicago, IL, USA
}

Received 9 November 2011; Accepted 9 November 2011

It was not too long ago that replying "Cytokines" to the question "What's your area of research?" elicited a puzzled look from most researchers. It is a clear sign of how much things have changed that, towards the end of 2011, a PubMed search with the word "cytokines" comes up with about half a million hits and a Google search generates a 4-million-plus list. It now seems that everybody, not just biomedical scientists, know something about cytokines. And yet there is so much more we need to understand.

Cytokines are small proteins that act as messengers between cells, carrying information that influences and determines a vast variety of functions, from directing embryonic development to maintaining homeostasis, from signaling to neurons to modulating bone structure [1]. But cytokines are most famous for their role as mediators and regulators of inflammatory responses [2]. With chronic inflammation being recognized as the main underlying pathophysiological mechanism of the majority of diseases affecting vast swaths of the world population, from infections to joint degeneration, from atherosclerosis to diabetes, cancer, autoimmunity and many more, it is no surprise that research into the role and regulation of cytokines, one of the most important family of inflammatory mediators, is on a constant rise. New cytokines are discovered, new functions for old ones unraveled, and new mechanisms of action clarified seemingly every day.

This special issue of TheScientificWorldJOURNAL includes reviews and primary research articles on two aspects of cytokine biology that are being recognized as playing critical roles in the regulation of inflammation and chronic pathologies. The first section of this special issue deals with the inflammasome and the interleukin (IL)-1 family of cytokines, whereas the second section discusses aspects of adipokines and adipose tissue biology.

Interleukin-1, one of the first cytokines to be described and cloned, is currently a pharmacological target for treatment of several conditions [3, 4]. However, despite having been known for decades, several aspects of IL-1 biology are still puzzling. In particular, the mechanisms leading to activation by proteolytic cleavage and subsequent release of the mature and active moiety of one of the two types of IL- 1 , IL- $1 \beta$, have been under scrutiny since the molecule was discovered and initially characterized as lacking the typical signal sequence of secreted proteins [5]. We now understand that a complex multimolecular machinery known as the inflammasome activates caspase-1, the main enzyme responsible for cleavage of pro-IL$1 \beta$ (and the structurally similar IL-18) into the active form [6]. Inflammasomes are also responsible for induction of pyroptosis, a recently described type of programmed cell death that clears the body of infected cells [7]. Two review articles in this special issue of TheScientificWorldJOURNAL describe the involvement of the inflammasome in disease. The paper by M. Sahoo and colleagues reviews the mechanisms leading to activation of the inflammasome, release of IL-1 $\beta$ and IL-18, and induction of pyroptosis in the context of bacterial infections, whereas the paper by D. Lissner and B. Siegmund reviews the multifaceted involvement of the inflammasome in the pathogenesis of inflammatory bowel disease, a chronic inflammatory condition 
of the gastrointestinal tract. The third paper of the first section, by A.-M. Balau et al., presents results of primary research on one of the newest members of the IL-1 family of cytokines, IL-37, and its involvement in acute inflammatory liver disease. As a whole, the three papers of this section highlight the necessity to learn more about the complexities of the inflammasome and the ever-expanding family of IL-1-like cytokines before targeted therapeutic intervention can be developed.

The second section of this special issue contains papers on adipose tissue and adipokines. Despite being considered for a long time as a mostly inert tissue, simply devoted to energy storage, adipose tissue is now clearly identified as a pivotal contributor to body homeostasis and to the propensity to develop diseases such as diabetes, atherosclerosis, cancer and many more [8]. The worldwide epidemic of obesity is pushing scientists to better understand the connection between adipose tissue biology and mechanisms of disease. Adipokines are a family of cytokines produced by cells present in adipose tissue, that is, adipocytes and infiltrating inflammatory cells [9]. Adipokines, including leptin, adiponectin, IL-6 and many more, regulate several aspects of biology such as food intake, glucose and lipid metabolism, angiogenesis and so forth. Evidence is rapidly accumulating that adipokines and adipose tissue are critical determinants of disease susceptibility and that obesity increases morbidity through dysregulation of adipose tissue function and adipokine production. The paper by M. Scotece and colleagues in this issue of The Scientific World discusses evidence implicating adipokines in the regulation of the inflammatory response in rheumatic diseases, thus linking adipokine production to autoimmune conditions. Two primary research articles also belong to this second section. The paper by M.-H. Nguyen et al. investigates the involvement of the adipokine leptin and of its receptor in regulating tissue regeneration following muscle damage, while the paper by L. TussingHumphreys and colleagues evaluates regulation of hepcidin, a major modulator of iron metabolism, in adipose tissue. These three papers together report on the far-reaching effects of molecules mostly produced by adipose tissue and the potential unexpected consequences of adipose tissue dysfunction in obesity.

As disparate as the topics of the two sections of this special issue appear to be, the IL-1 family of cytokines, the inflammasome, adipokines, and adipose tissue biology are actually connected, with molecules and mediators discussed in the first section emerging as important modulators of adipose tissue biology, while adipokines and adipocytes presented in section two modulate production and activity of the protagonists of section one [10]. It seems that cytokines are breaking the barriers between disciplines that traditionally dealt with one tissue, cell, or response at a time and are pulling the body back together as a whole.

\section{REFERENCES}

[1] A. W. Thomson and M. T. Lotze, The Cytokine Handbook, Academic Press, 2003.

[2] B. B. Aggarwal, S. Krishnan, and S. Guha, Inflammation, Lifestyle and Chronic Diseases: the Silent Link, CRC Press, 2011.

[3] C. A. Dinarello, "Interleukin-1 in the pathogenesis and treatment of inflammatory diseases," Blood, vol. 117, no. 14, pp. 3720-3732, 2011.

[4] C. A. Dinarello, N. P. Goldin, and S. M. Wolff, "Demonstration and characterization of two distinct human leukocytic pyrogens," Journal of Experimental Medicine, vol. 139, no. 6, pp. 1369-1381, 1974.

[5] C. A. Dinarello, "Blocking interleukin- $1 \beta$ in acute and chronic autoinflammatory diseases," Journal of Internal Medicine, vol. 269, no. 1, pp. 16-28, 2011.

[6] F. L. van de Veerdonk, M. G. Netea, C. A. Dinarello, and L. A.B. Joosten, "Inflammasome activation and IL-1 $\beta$ and IL-18 processing during infection," Trends in Immunology, vol. 32, no. 3, pp. 110-116, 2011.

[7] M. Lamkanfi and V. M. Dixit, "Modulation of inflammasome pathways by bacterial and viral pathogens," Journal of Immunology, vol. 187, no. 2, pp. 596-602, 2011.

[8] G. Fantuzzi and T. Mazzone, Adipose Tissue and Adipokines in Health and Disease, Humana Press, 2007.

[9] G. Fantuzzi, "Adipose tissue, adipokines, and inflammation," Journal of Allergy and Clinical Immunology, vol. 115, no. 5, pp. 911-920, 2005.

[10] D. De Nardo and E. Latz, "NLRP3 inflammasomes link inflammation and metabolic disease," Trends in Immunology, vol. 32, no. 8, pp. 373-379, 2011. 
This article should be cited as follows:

Giamila Fantuzzi, "Cytokines: Pulling the Body Together as a Whole," TheScientificWorldJOURNAL, vol. 11, pp. 2506-2508, 2011. 


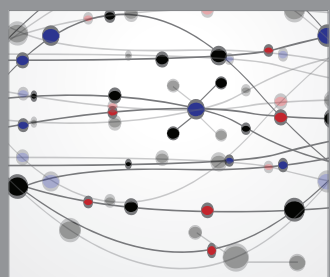

The Scientific World Journal
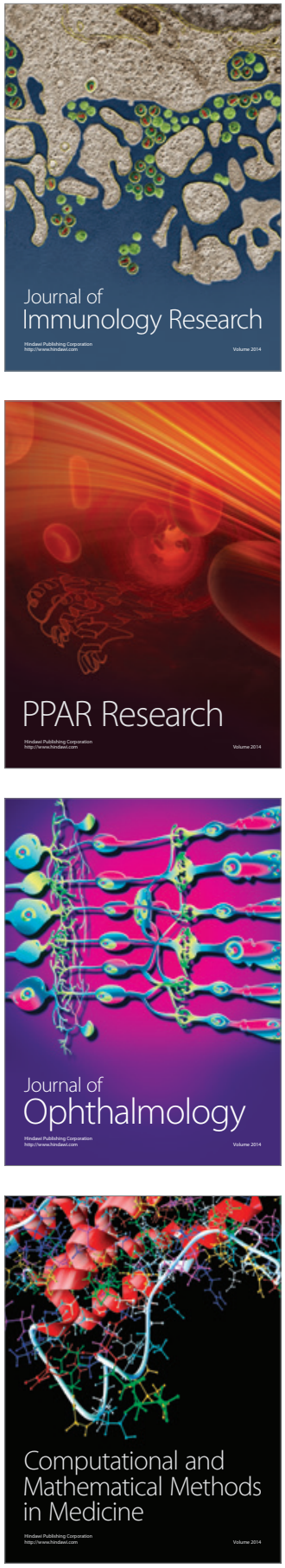

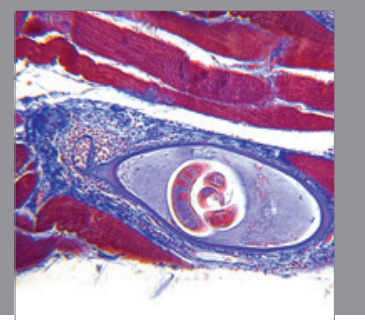

Gastroenterology

Research and Practice
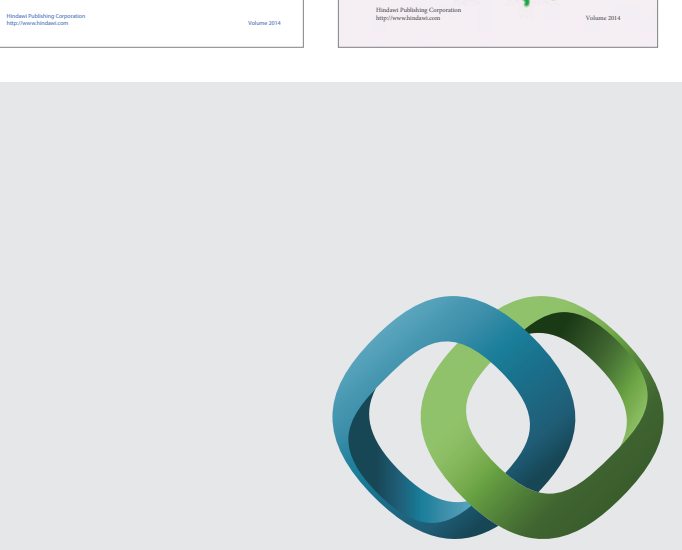

\section{Hindawi}

Submit your manuscripts at

http://www.hindawi.com
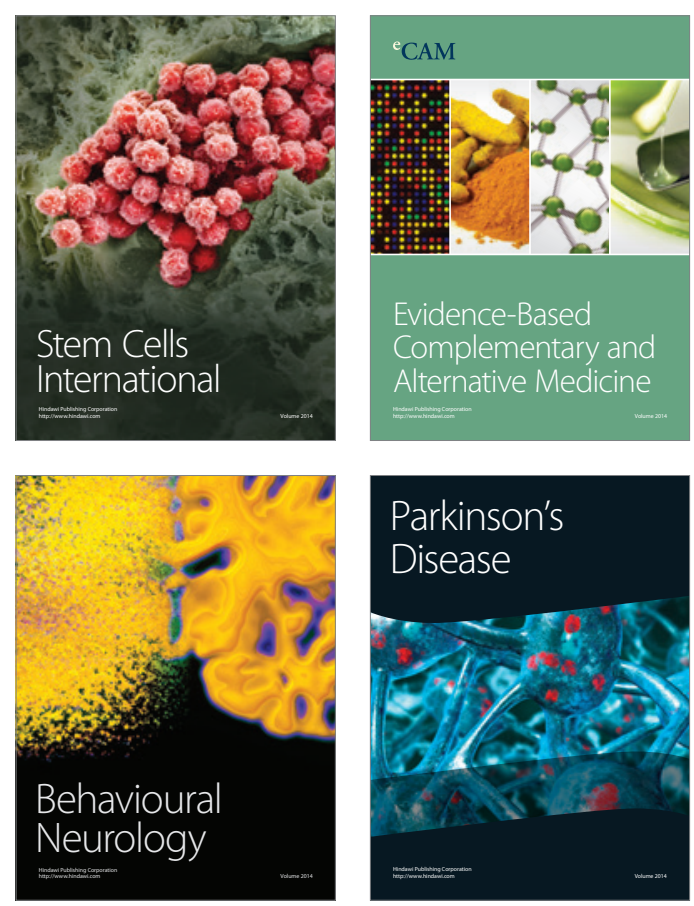

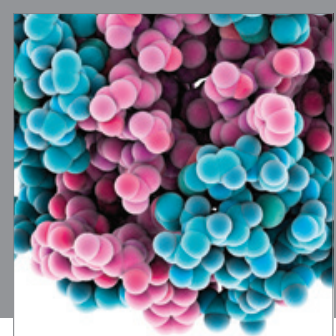

Journal of
Diabetes Research

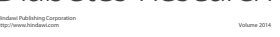

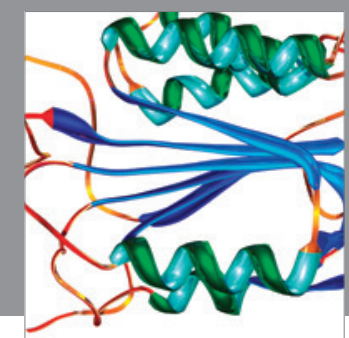

Disease Markers
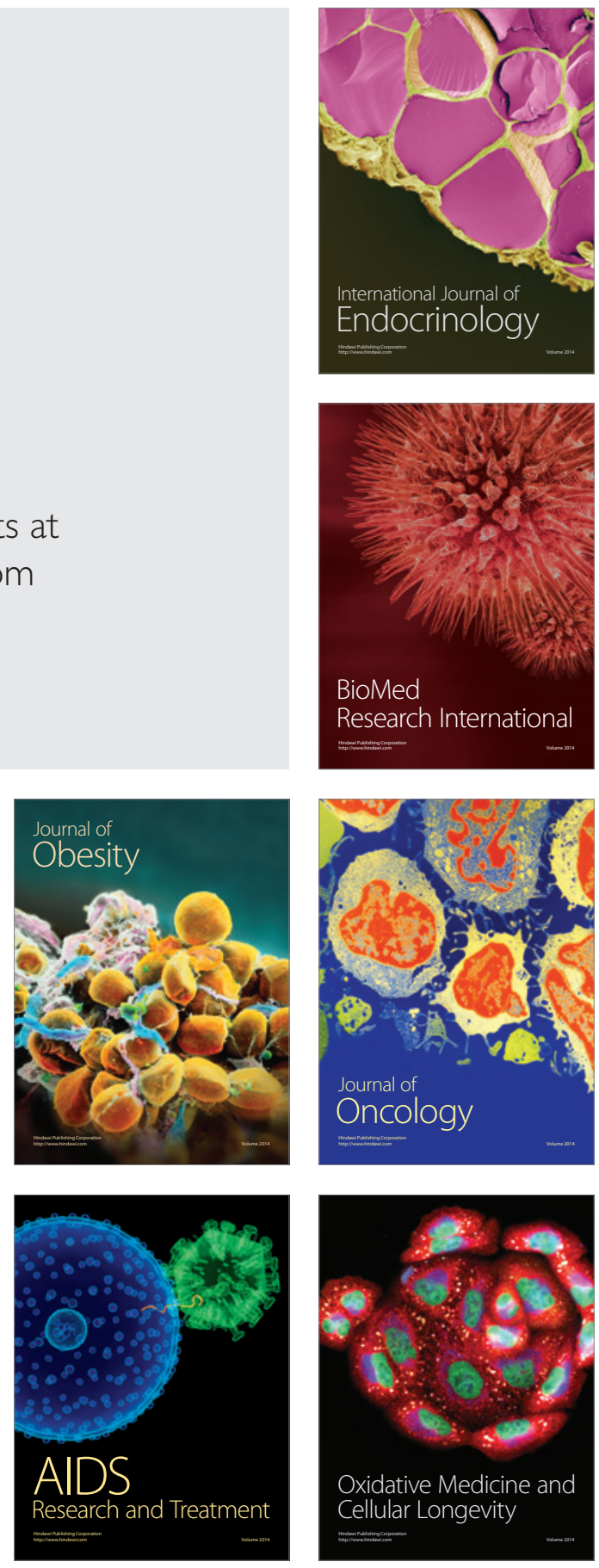